\title{
Communication
}

\section{Arginine52 Controls the Photoisomerization Process in Photoactive Yellow Protein}

Gerrit Groenhof, Lars V. Schfer, Martial Boggio-Pasqua, Helmut Grubmller, and Michael A. Robb J. Am. Chem. Soc., 2008, 130 (11), 3250-3251• DOI: 10.1021/ja078024u • Publication Date (Web): 23 February 2008

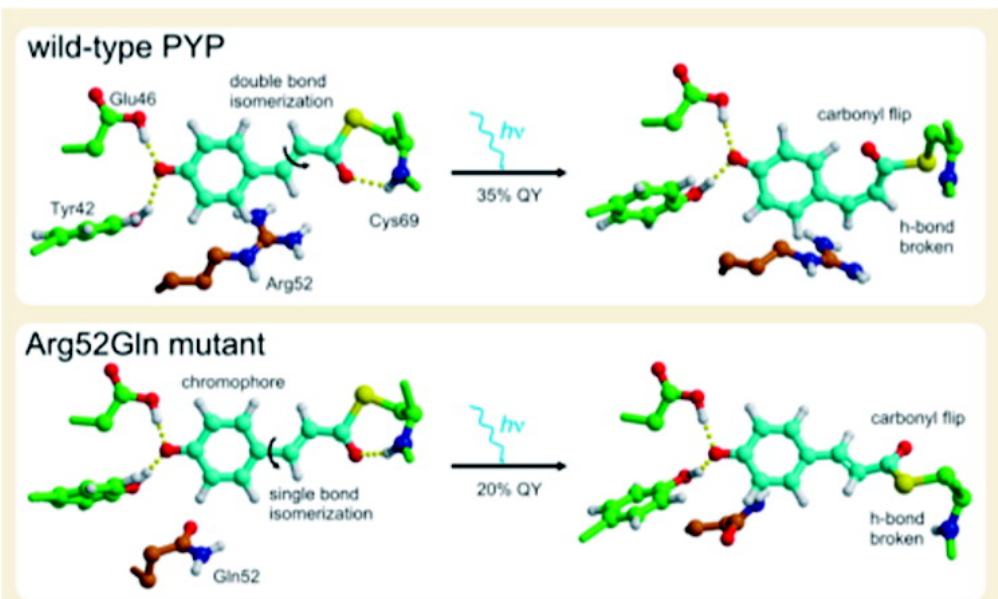

\section{More About This Article}

Additional resources and features associated with this article are available within the HTML version:

- Supporting Information

- $\quad$ Links to the 2 articles that cite this article, as of the time of this article download

- $\quad$ Access to high resolution figures

- $\quad$ Links to articles and content related to this article

- $\quad$ Copyright permission to reproduce figures and/or text from this article

\section{View the Full Text HTML}

\section{ACS Publications}




\title{
Arginine52 Controls the Photoisomerization Process in Photoactive Yellow Protein
}

\author{
Gerrit Groenhof, ${ }^{*}{ }^{\dagger}$ Lars V. Schäfer,${ }^{\dagger}$ Martial Boggio-Pasqua, ${ }^{\dagger}$ Helmut Grubmüller,${ }^{\dagger}$ and \\ Michael A. Robb§ \\ Department of Theoretical and Computational Biophysics, Max-Planck-Institute for Biophysical Chemistry, \\ Am Fassberg 11, 37077 Göttingen, Germany, Laboratoire de Chimie et Physique Quantiques, IRSAMC, \\ Université Paul Sabatier, 118 Route de Narbonne, 31062 Toulouse, France, and Department of Chemistry, \\ Imperial College London, London SW7 2AZ, United Kingdom
}

Received October 19, 2007; E-mail: ggroenh@gwdg.de

Photoactive yellow protein (PYP) is believed to be the primary photoreceptor for the photo avoidance response of the salt-tolerant bacterium Halorhodospira halophila. PYP contains a deprotonated 4-hydroxy-cinnamic acid (or $p$-coumaric acid, pca) chromophore linked covalently to the $\gamma$-sulfur of Cys69 via a thioester bond (Figure 1). ${ }^{1}$ Upon absorbing a blue-light photon, PYP enters a fully reversible photocycle involving several intermediates on a time scale spanning from a few hundred femtoseconds to seconds. ${ }^{2}$ In previous work we have used mixed quantum/classical (QM/MM) molecular dynamics (MD) simulations to reveal the detailed sequence of structural changes that follows photoabsorption in wild-type PYP. ${ }^{3}$ The first step is the trans-to-cis photoisomerization of the chromophore, followed by a flip of the thioester carbonyl group by $180^{\circ}$. We found that in the protein environment the isomerization is enhanced by a preferential electrostatic stabilization of the chromophore's excited state by the guanidinium group of Arg52, located just above the negatively charged chromophore ring. However, mutants in which the arginine has been replaced by an electronically neutral amino acid can still enter the photocycle, albeit with a lower rate and quantum yield. ${ }^{4}$ These findings indicate that Arg52 is important but not essential for photoactivation.

To elucidate the role of this arginine in the activation process in more detail, we have performed a new series of excited-state dynamics simulations of the Arg52Gln (R52Q) mutant of PYP (entry $2 \mathrm{D} 02$ of the PDB). ${ }^{5}$ The results of these simulations demonstrate that in the mutant, the primary events after photoexcitation are different from those in the wild-type. First, the predominant excited-state decay channel in the mutant involves isomerization of a single bond in the chromophore $\left(\mathrm{C}_{3}-\mathrm{C}_{1^{\prime}}\right.$, Figure 2 ), rather than the double bond $\left(\mathrm{C}_{2}=\mathrm{C}_{3}\right)$. Although single bond isomerization does not result in the formation of a new chromophore isomer, a $180^{\circ}$ flip of the thioester carbonyl oxygen atom was observed in 7 out of 40 trajectories. Together with the experimental observation that the mutant is photoactive, this suggests that the crucial event in the photoactivation is the oxygen flip, rather than the double bond isomerization. The second difference is that the photochemical process is slower in the mutant than in the wildtype, in agreement with recent measurements. ${ }^{6}$

As in our previous work, QM/MM MD simulations were carried out at the CASSCF $(6,6) / 3-21 \mathrm{G}$ level of theory, with diabatic surface hopping between the excited $\left(\mathrm{S}_{1}\right)$ and ground state $\left(\mathrm{S}_{0}\right)$ potential energy surfaces. In total, 40 excited-state simulations were initiated from different frames of a 250 ps ground state-trajectory (see Supporting Information for details). In these simulations, an excited-

\footnotetext{
$\dagger$ Max-Planck-Institute for Biophysical Chemistry.

\# Université Paul Sabatier.

§ Imperial College London.
}

state lifetime between 155 and 1438 fs was observed (Supporting Information, Table S1, third column). A simple exponential fit yields a fluorescence decay time of $\tau=400 \pm 100 \mathrm{fs}$. The fluorescence lifetime is longer in the R52Q mutant than in the wild-type simulations $^{3}$ ( $\tau=200 \mathrm{fs}$ ), a difference that was also observed in recent experiments. ${ }^{6}$

In contrast to wild-type PYP, the photoisomerization takes place predominantly around the single $\mathrm{C}_{3}-\mathrm{C}_{1}$, bond rather than the double $\mathrm{C}_{2}=\mathrm{C}_{3}$ bond. Figure 2 shows a plot of the torsion angles in the tail of the chromophore during a single bond photoisomerization trajectory. There are three distinct phases: (I) evolution on $\mathrm{S}_{0}$, (II) excitation and evolution on $S_{1}$, and (III) decay to $S_{0}$ at the surface crossing followed by evolution on the $S_{0}$ surface. Immediately after the excitation, the chromophore decays (via bond length relaxation) from the Franck-Condon region into a nearby local trans minimum on $S_{1}$. After 0.3 ps in $S_{1}$, a barrier separating this local minimum from a twisted $\mathrm{S}_{1}$ minimum is crossed and the chromophore relaxes further (via rotation of the single $\mathrm{C}_{3}-\mathrm{C}_{1^{\prime}}$ bond (torsion $a$, Figure 2, black line) by $90^{\circ}$ ) into a twisted $\mathrm{S}_{1}$ minimum conformation. Concerted rotation takes place around the $\mathrm{C}_{\beta}-\mathrm{S}_{\gamma}$ bond (Figure 2, yellow line). These simultaneous rotations in opposite directions avoid a large out-of-plane motion of the phenoxy ring. During the process, the hydrogen bond network connecting the chromophore's phenoxy atom $\left(\mathrm{O}_{4^{\prime}}\right)$ to Tyr42 and Glu46 remains intact (Figure 1). On average, it takes about 300 fs to cross the barrier and reach the twisted minimum. This activation barrier involves breaking the hydrogen bond between the chromophore's carbonyl $\mathrm{C}_{1}=\mathrm{O}_{1}$ and the Cys69 backbone amino group upon rotation of the $\mathrm{C}_{\beta}-\mathrm{S}_{\gamma}$ bond.

The system oscillates around the single-bond twisted minimum until the surface crossing seam is encountered and a surface hop takes the system back to the ground state. It takes more time to reach the conical intersection hyperline in the mutant. The $S_{1} / S_{0}$ seam lies higher in energy and is therefore more difficult to reach than in the wild-type, in which passage of the seam is facilitated due to electrostatic stabilization of the excited-state charge distribution by the positively charged guanidinium group of Arg52.

In 32 out of 40 simulations (80\%), the broken hydrogen bond between the carbonyl $\mathrm{C}_{1}=\mathrm{O}_{1}$ and the Cys69 backbone amino group is reformed quickly after the radiationless transition, leading to the original ground-state configuration. In eight trajectories (20\%), this hydrogen bond is not restored. Instead, the carbonyl group flips over completely to the other side of the chromophore after the hop to $S_{0}$ (Figure 2). Figure 1c shows the geometry of the active site after this carbonyl flip. Because of the concerted bond rotations (see above), the excited-state dynamics of the carbonyl flip resembles the bicycle pedal mechanism ${ }^{7}$ with the $\mathrm{C}_{1}-\mathrm{C}_{2}$ bond forming the crank axis and the $\mathrm{C}_{3}-\mathrm{C}_{1^{\prime}}$ and $\mathrm{C}_{\beta}-\mathrm{S}_{\gamma}$ bonds the pedals. 


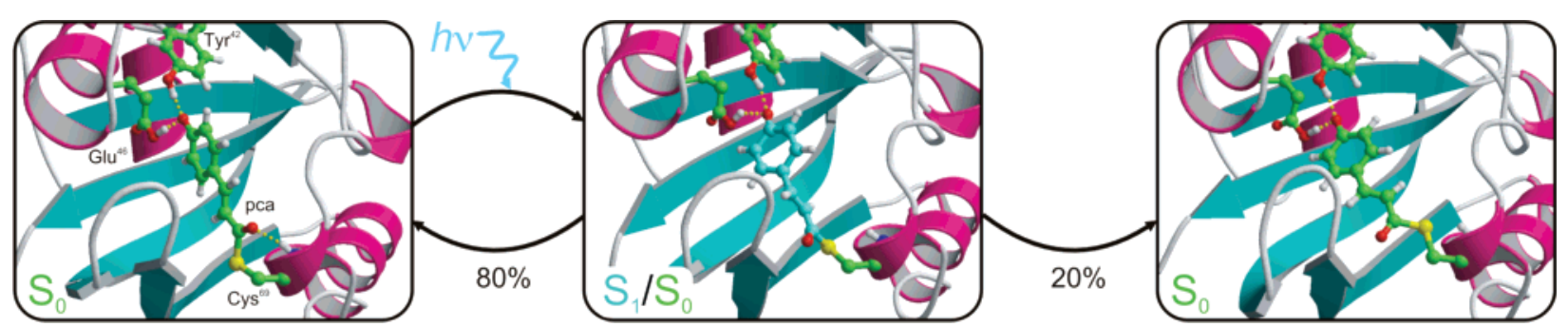

Figure 1. Snapshots from a photoisomerization trajectory showing the chromophore (pca) in the active site of the protein. The first shapshot is at the excitation, the second shows the configuration at the $S_{1} / S_{0}$ conical intersection, and the third is after the relaxation into a local $S_{0}$ minimum that has the carbonyl oxygen flipped. The latter occurs with a quantum yield of $20 \%$. The other trajectories return to the original configuration (first snapshot).

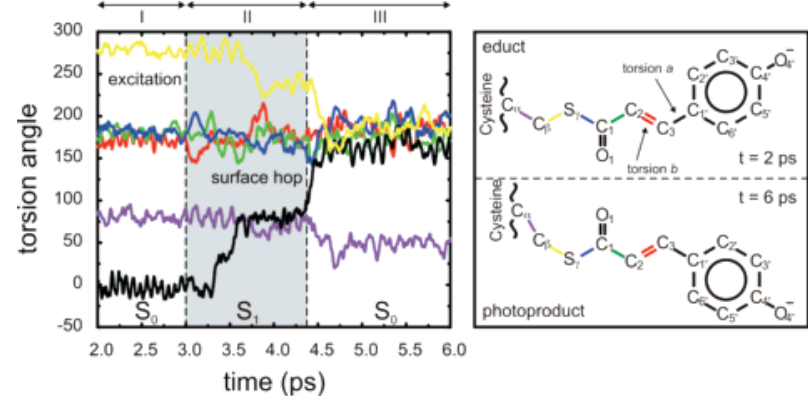

Figure 2. Behavior of the torsion angles in the tail of the chromophore during a photoisomerization trajectory: $\mathrm{C}_{\alpha}-\mathrm{C}_{\beta}$ (purple), $\mathrm{C}_{\beta}-\mathrm{S} \gamma$ (yellow), $\mathrm{S} \gamma-\mathrm{C}_{1}$ (blue), $\mathrm{C}_{1}-\mathrm{C}_{2}$ (green), $\mathrm{C}_{2}=\mathrm{C}_{3}$ (red), and $\mathrm{C}_{3}-\mathrm{C}_{1^{\prime}}$ (black). Stage $\mathrm{I}$, before photoexcitation; stage II, isomerization of torsion $a$ after photoexcitation to $\mathrm{S}_{1}$ (shaded area); and stage III, after decay to the ground state $\left(\mathrm{S}_{0}\right)$.

In one of the simulations, a double bond $\left(\mathrm{C}_{2}=\mathrm{C}_{3}\right)$ isomerization was observed. As in the wild-type, ${ }^{3}$ this isomerization also causes the hydrogen bond between $\mathrm{C}_{1}=\mathrm{O}_{1}$ and $\mathrm{N}-\mathrm{H}$ to break. This observation demonstrates that in the mutant the double bond photoisomerization channel is still accessible, albeit with a much lower probability than the single bond isomerization. Animations are available as Supporting Information.

Statistically the number of trajectories is small but nevertheless yields a consistent picture of the role of Arg52 on the photoisomerization process in PYP. Arg52 controls which bond isomerizes upon photoexcitation. Without its positive charge in the vicinity of the chromophore, the isomerization involving single bond rotation is favored over double bond isomerization. This observation is supported by the recent coupled-cluster gas-phase computations of Gromov et al. ${ }^{8}$ The charge distributions of the single and double bond twisted $S_{1}$ minima are different. In the single bond twisted structure, there is a net negative charge on the alkene moiety of the chromophore, whereas in the double bond twisted structure, the negative charge is located on the phenolate ring. ${ }^{8}$ Thus, in the wild-type protein environment, where the positive charge of Arg52 is located right above the phenolate, the double bond twisted $S_{1}$ minimum is electrostatically stabilized relative to the single bond twisted structure. In contrast, the lack of this positive charge in the mutant resembles the situation in vacuum, where single bond rotation is preferred. ${ }^{3}$ Furthermore, as Gromov et al. have also shown, ${ }^{8}$ the gap between $S_{1}$ and $S_{0}$ is smaller in the double bond than in the single bond twisted minimum, accounting for the slower excited-state decay observed for the mutant.

The flip of the carbonyl $\left(\mathrm{C}_{1}=\mathrm{O}_{1}\right)$ leads to an intermediate that remains stable for nanoseconds in subsequent force-field simulations. We therefore suggest that this flip and the concomitant break of the hydrogen bond between the carbonyl oxygen of the chromophore and the backbone amino group is the crucial step in the photoactivation of PYP. Rupture of this hydrogen bond has also been suggested by others to be the key step to enter the photocycle. ${ }^{9,10}$ Furthermore, the experimentally observed quantum yield of the R52Q mutant $(21 \pm 8 \%)^{4}$ matches closely to the carbonyl flip quantum yield in our simulations (20\% including the flip caused by the double bond isomerization).

Thus, we think that both single and double bond photoisomerization can lead to a photoreactive intermediate that is required to enter the photocycle. The efficiency of the double bond isomerization in the wild-type (30\%) is higher than that of the single bond in the mutant (20\%), highlighting the importance of Arg52 for the photoactivation. Furthermore, the arginine also reduces the excitedstate lifetime, suggesting it could play a role in photoprotection as well. How the broken hydrogen bond between $\mathrm{C}_{1}=\mathrm{O}_{1}$ and the Cys69 aminogroup eventually leads to large-scale conformational changes in PYP is still an open question. However, addressing this issue is a formidable task that goes beyond the scope of the current work.

Acknowledgment. Support from the DFG (Grant SFB 755) is thankfully acknowledged. We thank Klaas Hellingwerf for critically reading the manuscript and valuable comments.

Supporting Information Available: Details of the QM/MM scheme, surface hopping algorithm, protein model, and animations (MPEG1) of photoisomerization trajectories. This material is available free of charge via the Internet at http://pubs.acs.org.

\section{References}

(1) Hoff, W. D.; Dux, P.; Hard, K.; Devreese, B.; Nugteren-Roodzant, I. M.; Crielaard, W.; Boelens, R.; Kaptein, R.; van Beeumen, J.; Hellingwerf, K. J. Biochemistry 1994, 33, 13959-13962.

(2) Hellingwerf, K. J.; Hendriks, J.; Gensch, T. J. Phys. Chem. A 2003, 107 , $1082-1094$

(3) Groenhof, G.; Bouxin-Cademartory, M.; Hess, B.; de Visser, S. P.; Berendsen, H. J. C.; Olivucci, M.; Mark, A. E.; Robb, M. A. J. Am. Chem. Soc. 2004, 124, 4228-4232.

(4) Takeshita, K.; Imamoto, Y.; Kataoka, M.; Mihara, K.; Tokunaga, F.; Terazima, M. Biophys. J. 2002, 83, 1567-1577.

(5) Shimizu, N.; Kamikubo, H.; Yamazaki, Y.; Imamoto, Y.; Kataoka, M. Biochemistry 2006, 45, 3542-3547.

(6) (a) Changenet-Barret, P.; Plaza, P.; Martin, M. M.; Chosrowjan, H.; Taniguchi, S.; Mataga, N.; Imamoto, Y.; Kataoka, M. Chem. Phys. Lett. 2007, 434, 320-325. (b) Mataga, N.; Chosrowjan, H.; Taniguchi, S. J. Photochem. Photobiol. C. 2004, 5, 155-168.

(7) Warshel, A. Nature 1976, 260, 679-683.

(8) Gromov, E. V.; Burghardt, I.; Hynes, J. T.; Köppel, H.; Cederbaum, L. S. J. Photochem. Photobiol. A. 2007, 190, 241-257.

(9) Van Wilderen, L. J. G. W.; Van der Horst, M. A.; Van Stokkum, I. H. M.; Hellingwerf, K. J.; Van Grondelle, R.; Groot, M. L. Proc. Natl. Acad. Sci. U.S.A. 2006, 103, 15050-15055.

(10) Cordfunke, R.; Kort, R.; Pierik, A.; Gobets, B.; Koomen, G. J.; Verhoeven, J. W.; Hellingwerf, K. J. Proc. Natl. Acad. Sci. U.S.A. 1998, 95, $7396-7401$.

JA078024U 